\title{
Deletion of the KIT gene is associated with liver metastasis and poor prognosis in patients with gastrointestinal stromal tumor in the stomach
}

\author{
${\text { SONGDE } \text { CHO }^{1} \text {, YASUHIKO KITADAI }}^{1}$, SHIGETO YOSHIDA ${ }^{1}$, SHINJI TANAKA ${ }^{2}$, \\ MASAHARU YOSHIHARA $^{3}$, KAZUHIRO YOSHIDA $^{4}$ and KAZUAKI CHAYAMA ${ }^{1}$
}

\begin{abstract}
${ }^{1}$ Department of Medicine and Molecular Science, Hiroshima University Graduate School of Biomedical Sciences, 1-2-3 Kasumi, Minami-ku, Hiroshima 734-8551; ${ }^{2}$ Department of Endoscopy, Hiroshima University Hospital, Hiroshima; ${ }^{3}$ Health Service Center, Hiroshima University, Higashi-Hiroshima; ${ }^{4}$ Department of Oncology, Research Institute for Radiation Biology and Medicine, Hiroshima University, Hiroshima, Japan
\end{abstract}

Received December 28, 2005; Accepted February 21, 2006

\begin{abstract}
The goal of this study was to investigate the association of mutations in the KIT gene and the plateletderived growth factor receptor $\alpha$ (PDGFRA) gene with clinicopathological features of patients with gastrointestinal stromal tumor (GIST) localized in the stomach. We evaluated 56 gastric GISTs for KIT and PDGFRA mutations. DNA was extracted from paraffin-embedded tumor specimens, and exons 9, 11, 13 and 17 of the KIT gene and exons 12 and 18 of the PDGFRA gene were amplified by polymerase chain reaction and sequenced. The genetic features were then compared with the clinicopathological features. Immunohistochemistry was performed for KIT, CD34, Ki-67 (as a marker of cell proliferation) and CD31 (as a marker of microvessel density), and apoptosis was assessed by in situ DNA nick-end labeling. Thirty-four $(61 \%)$ of the 56 GISTs had a mutation in exon 11 of KIT, and 2 (4\%) had a mutation in exon 13 of KIT. Deletions in exon 11 of KIT were the most common mutation encountered in the present study. No mutations were found in exon 9 or 17 of KIT. Six of the 20 GISTs lacking KIT mutations had a mutation in exon 18 of PDGFRA, and 1 had a mutation in exon 12 of PDGFRA. The KIT mutation-positive GISTs showed more frequent liver metastases and higher mortality than KIT mutation-negative GISTs. Our data indicate that KIT mutations, especially deletions in exon 11, are markers of poor prognosis for gastric GISTs.
\end{abstract}

Correspondence to: Dr Yasuhiko Kitadai, Department of Medicine and Molecular Science, Hiroshima University Graduate School of Biomedical Sciences, 1-2-3 Kasumi, Minami-ku, Hiroshima 734-8551, Japan

E-mail: kitadai@hiroshima-u.ac.jp

Key words: gastrointestinal stromal tumor, KIT, platelet-derived growth factor receptor $\alpha$, mutation, deletion, stomach

\section{Introduction}

Gastrointestinal stromal tumor (GIST) is the most common mesenchymal tumor of the human gastrointestinal tract. Although stromal tumors can occur throughout the GI tract from the esophagus to the rectum, they occur most frequently in the stomach (1), less frequently in the small intestine, and least often at other sites. GISTs differ from other mesenchymal tumors histologically, immunohistochemically and genetically (2). Only GISTs express KIT, a KIT protooncogene protein, and positivity for KIT has been reported in $89-100 \%$ of all GISTs $(3,4)$. Sequencing of the KIT gene has revealed activating mutations in many GISTs (5). These KIT gene mutations have not been observed in other mesenchymal tumors, such as leiomyomas or leiomyosarcomas $(2,6)$. Recently, it has been reported that a subset of GISTs that lacked KIT mutations had activating mutations in the related receptor tyrosine kinase, platelet-derived growth factor receptor $\alpha$ (PDGFRA) (7).

At present, the best treatment for localized GIST is complete surgical excision; however, relapse is not unusual. Prediction of relapse and metastasis of GISTs on the basis of clinicopathological features is often difficult. In addition, non-operable GISTs, such as metastatic or invasive GISTs, are typically resistant to conventional chemotherapies. Recently, imatinib mesylate (STI571), which was developed for treatment of chronic myeloid leukemia (CML) $(8,9)$, was found to be useful for treatment of advanced GISTs (10-13). Although GISTs are less common than gastrointestinal carcinomas or lymphomas, they have received attention as a model system for development of molecular therapies.

It has been reported that GISTs with KIT mutations have poorer prognoses than those lacking these mutations $(2,6,14-16)$. In contrast, there are reports that KIT mutations are not associated with malignancy $(17,18)$. Therefore, the prognostic significance of KIT mutations remains controversial.

In the present study, we examined the correlation of alterations in exons $9,11,13$ and 17 of the KIT gene and in exons 12 and 18 of the PDGFRA gene in gastric GISTs with cell proliferation, angiogenesis and apoptosis. We also 
investigated whether alterations in KIT are important prognostic markers in gastric GISTs.

\section{Patients and methods}

Patients and tumor specimens. We retrospectively analyzed the clinical records of 74 patients who underwent curative surgical resection for mesenchymal tumor of the stomach at Hiroshima University Hospital during the period of 1980-2000. Mesenchymal tumors were identified as GISTs on the basis of positive immunohistochemical staining for KIT and/or CD34. With this criterion, 62 of the 74 tumors (84\%) were identified as GISTs. All patients presented with a solitary tumor. Of the 62 patients, complete outcome data were not available for 6 patients, who were therefore excluded from the analyses. None of the remaining 56 patients was treated with STI571 before or after surgery. For strict privacy protection, identifying information for all samples was removed before analysis; the procedure was in accordance with the Japanese Government's Ethical Guidelines for Human Genome/Gene Research.

Immunohistochemistry. Expression of KIT, CD34, Ki-67 and CD31 was analyzed by immunohistochemistry (Fig. 1A-D). A representative tissue block was collected for each case, and immunohistochemistry was performed on $4-\mu \mathrm{m}$ thick sections of formalin-fixed, paraffin-embedded tissues cut from these blocks using the Envision system (Dako Cytomation, Carpinteria, CA) for KIT and CD34 or the LSAB2 kit (Dako Cytomation) for Ki-67 and CD31.

All tissue sections for immunohistochemistry were deparaffinized in xylene and rehydrated with graded ethanol washes $(100-70 \%)$. Sections were then treated with pronase (trypsin; Difco, Detroit, MI) for $30 \mathrm{~min}$ at room temperature prior to CD34 and CD31 immunostaining and treated by microwave for 15 min prior to Ki-67 immunostaining. Sections were not treated before KIT immunostaining. Endogenous peroxidase activity was blocked with hydrogen peroxide for $10 \mathrm{~min}$ at room temperature, and sections were incubated with goat serum for $20 \mathrm{~min}$ at room temperature to block non-specific binding. The primary antibodies used were anti-KIT (diluted 1:50; Dako Cytomation, Kyoto, Japan), anti-CD34 (Histofine, Tokyo, Japan), anti-Ki-67 (MIB-1, diluted 1:50; Dako Cytomation, Glostrup, Denmark) and anti-CD31 (Dako Cytomation, Carpinteria, CA). The primary antibody was applied for $30 \mathrm{~min}$ at room temperature for KIT, $1 \mathrm{~h}$ at room temperature for Ki-67 and overnight at $4^{\circ} \mathrm{C}$ for CD34 and CD31. 3,3'-Diaminobenzidine (Merck, Darmstadt, Germany) was used as the chromogen, and sections were counterstained with hematoxylin.

The Ki-67-labeling index (LI) was determined by light microscopy of the area with the greatest number of Ki-67positive cells. These areas were identified by scanning tumor sections at low power (x40 and x100). The Ki-67 LI was calculated as the percentage of positive cells detected in 1,000 tumor cells.

Microvessel density (MVD) was determined by light microscopy examination of CD31 staining in the area with the greatest number of capillaries and small venules. Areas of high vascularity were identified by scanning tumor sections at low power (x40 and x100). Vessels were counted in the 3 areas of a x400 field (x40 objective and x10 ocular; $0.189 \mathrm{~mm}^{2} /$ field) with the greatest neovascularization, and the average count was noted.

In situ DNA nick-end labeling. DNA strand breaks due to apoptosis were detected in situ by terminal deoxynucleotidyl transferase-mediated dUTP-biotin nick-end labeling (TUNEL). This is based on the specific binding of terminal deoxynucleotidyl transferase to 3 '-OH ends of DNA. We used this method to detect apoptotic cells in gastric GISTs in $4-\mu \mathrm{m}$ thick sections of formalin-fixed, paraffin-embedded tissue (ApopTag ${ }^{\circledR}$; Chemicon, Temecula, CA). In brief, paraffin sections were deparaffinized, rehydrated through a graded alcohol series (100-70\%), and washed with distilled water. The tissue was digested with $20 \mu \mathrm{g} / \mathrm{ml}$ proteinase $\mathrm{K}$ for $15 \mathrm{~min}$ at room temperature. Sections were then treated with $3 \%$ $\mathrm{H}_{2} \mathrm{O}_{2}$ in phosphate-buffered saline (PBS) for $5 \mathrm{~min}$ at room temperature and washed with distilled water. After prehybridization treatment, terminal deoxynucleotidyl transferase with digoxigenin-11-deoxyuridine triphosphate (dUTP) and deoxyadenosine triphosphate (dATP) were added to cover the sections, which were incubated in a humidified chamber for $60 \mathrm{~min}$ at $37^{\circ} \mathrm{C}$. The sections were then incubated for $30 \mathrm{~min}$ at room temperature with an anti-digoxigenin-antibodyperoxidase conjugate before staining with a 3,3'-diaminobenzidine- $\mathrm{H}_{2} \mathrm{O}_{2}$ solution. The sections were washed thoroughly with PBS between incubation steps and then counterstained with hematoxylin. The percentage of positive apoptotic cells among approximately 1,000 tumor cells was calculated.

Molecular analysis. Genomic DNA was extracted from formalin-fixed, paraffin-embedded GIST tissue. In all cases, exon 9 and exon 11 of the KIT gene were evaluated for mutations by polymerase chain reaction (PCR) amplification and direct sequencing of the amplification products. Samples from patients negative for both exon 9 and exon 11 mutations were then screened for exon 13 and exon 17 mutations by the same method. Samples negative for these KIT mutations were finally screened for exon 12 and exon 18 of the PDGFRA gene.

Exons 9, 11, 13 and 17 of KIT and exons 12 and 18 of PDGFRA were amplified by PCR with the following primer pairs: KIT exon 9, forward TTTGGAAAGCTAGTGGTTCA and reverse ATGGTAGACAGAGCCTAAAC; KIT exon 11, forward GATCTATTTTTCCCTTTCTC and reverse AGCC CCTGTTTCATACTGAC; KIT exon 13, forward GCTTG ACATCAGTTTGCCAG and reverse AAAGGCAGCTTGG ACACGGCTTTA; KIT exon 17, forward TTTCTCCTCCA ACCTAATAG and reverse CCTTTGCAGGACTGTCAAGC; PDGFRA exon 12, forward TCCAGTCACTGTGCTGCTTC and reverse GCAAGGGAAAAGGGAGTCTT; and PDGFRA exon 18, forward CAGGGGTGATGCTATATCAGC and reverse TAAAGTGTGGGAGGATGAGCC.

PCR product sizes were 238, 174, 193, 180, 260 and 235 bp respectively. PCR conditions were $94^{\circ} \mathrm{C}$ for $10 \mathrm{~min}$ followed by 50 cycles of $94^{\circ} \mathrm{C}$ for $1 \mathrm{~min}, 57^{\circ} \mathrm{C}$ for $1 \mathrm{~min}$, and $72^{\circ} \mathrm{C}$ for $1 \mathrm{~min}$ and a final cycle of $72^{\circ} \mathrm{C}$ for $10 \mathrm{~min}$. PCR products were separated by agarose gel electrophoresis, and visualized by UV transillumination. PCR products were purified with 

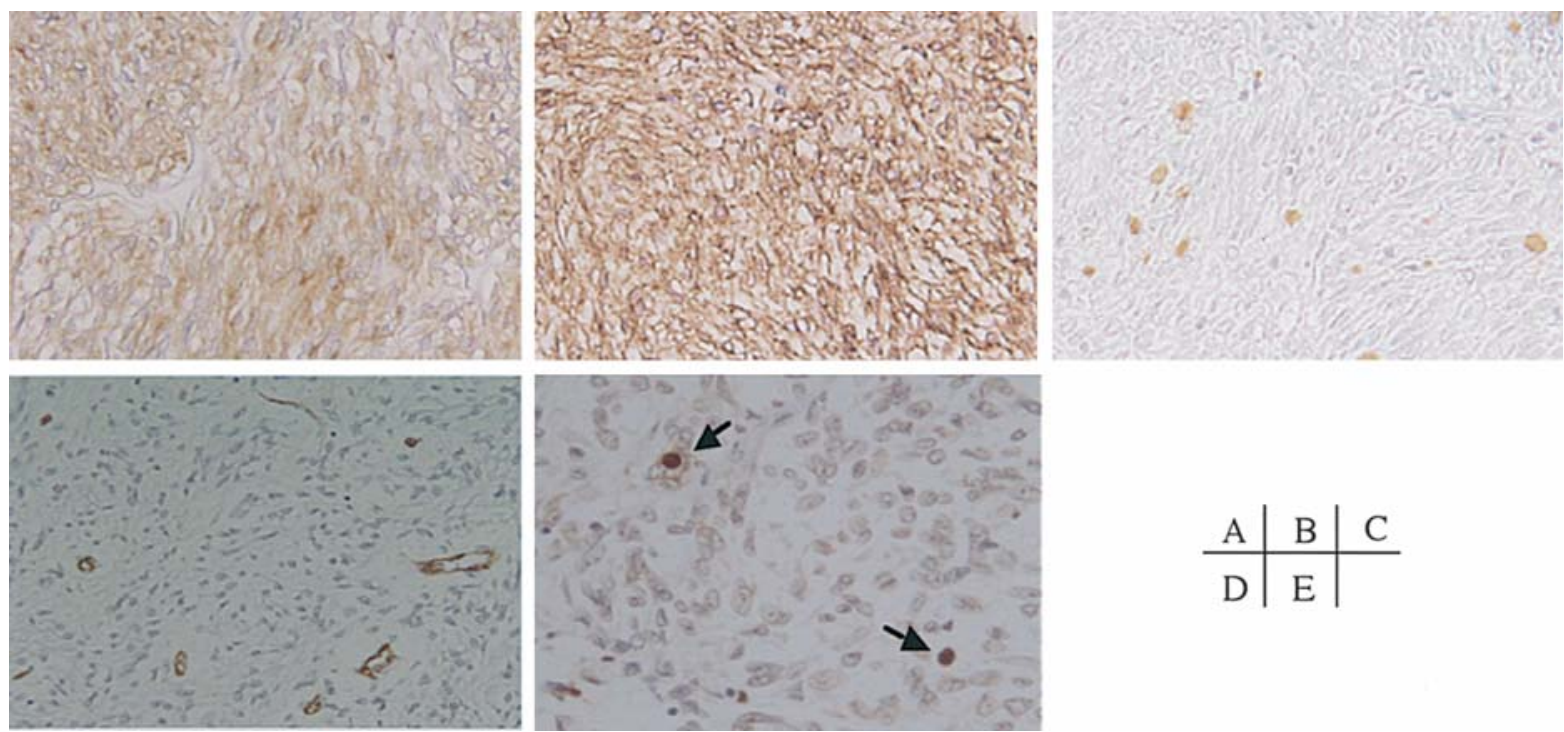

Figure 1. Immunohistochemical assessment of KIT and CD34 expression, proliferation, microvessel density, and apoptosis of gastric GISTs. (A) KIT and (B) CD34, most cells showed strong staining signals. (C) Ki-67 immunostaining. The number of Ki-67-positive cells among approximately 1,000 tumor cells was calculated as a percentage. (D) Immunohistochemical staining of CD31. Microvessels are visible as brown capillaries or small clusters that are distinct from the surrounding tissue. (E) Apoptotic cells stained by TUNEL. Positive nuclei are indicated by arrows.

(A)

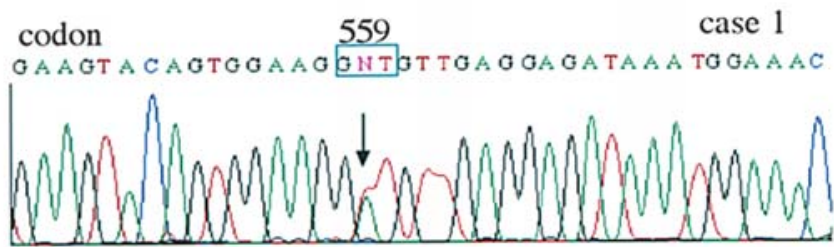

codon

$$
556557558
$$

GT NHG ANT NCANGNGHAGGTTGTTOAGAGATAAATGO
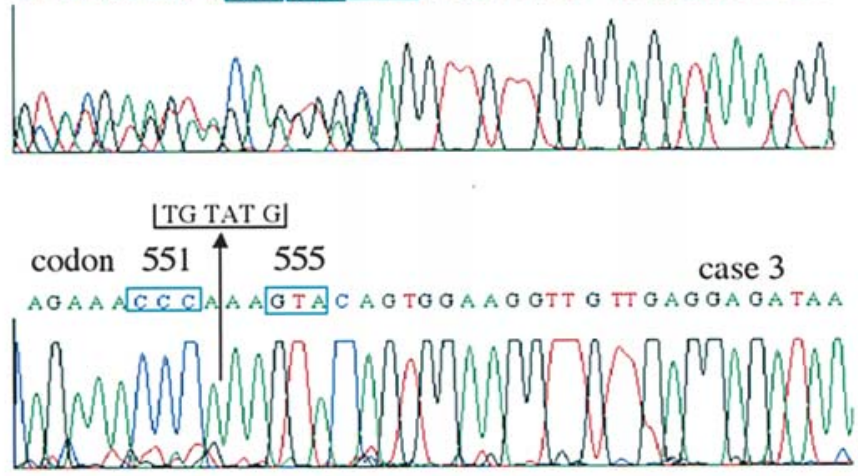

(B)

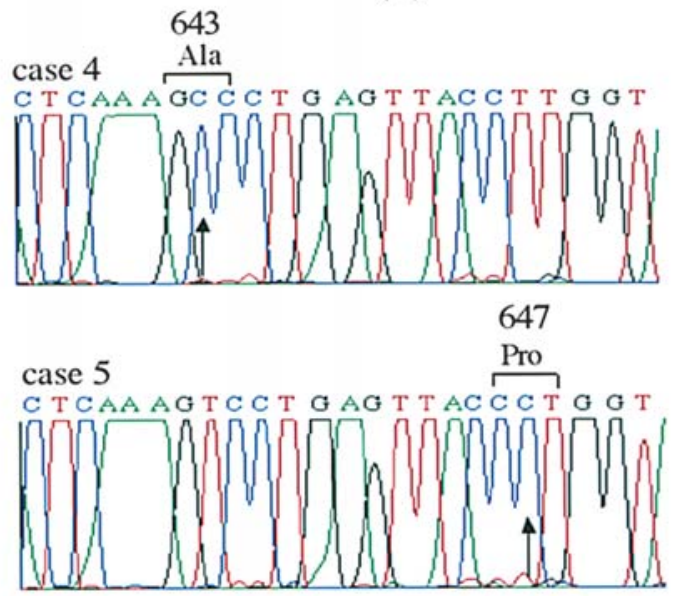

(C)

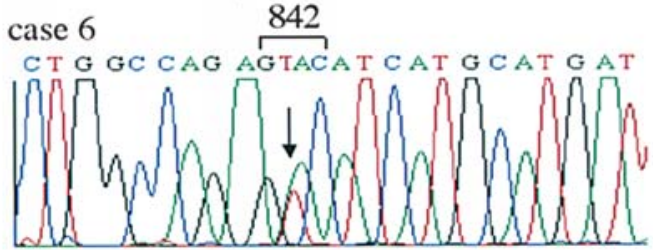

Figure 2. (A) Direct sequencing of mutant KIT exon 11 from GIST. All 3 cases shown were sequenced from the antisense direction. Case 1, arrow indicates heterozygous point mutation at the second nucleotide $(\mathrm{T} \rightarrow \mathrm{A})$ of codon 559 . Case 2, heterozygous in-frame deletion of 6 bp from codon 556 to 558 . Nucleotides (AGTGGA) are deleted at the site indicated by the double-headed arrow. Case 3, homozygous in-frame deletion of 6 bp. Nucleotides (TGTATG) are deleted at the site indicated by the arrow. (B) Direct sequencing of mutant KIT exon 13 from GIST. Case 4, arrow indicates a homozygous point mutation of the second nucleotide $(\mathrm{T} \rightarrow \mathrm{C}$ ) of codon 643, resulting in Val643Ala. Case 5, arrow indicates a homozygous point mutation of the second nucleotide ( $\rightarrow \mathrm{C}$ ) of codon 647, resulting in Leu647Pro. (C) Direct sequencing of mutant PDGFRA exon 18 from GIST. Case 6, arrow indicates heterozygous point mutation at the second nucleotide $(\mathrm{A} \rightarrow \mathrm{T})$ of codon 842 .

the DNA and Gel Band Purification kit (Amersham, Buckinghamshire, UK) before sequencing. KIT and PDGFRA were sequenced in both the forward and reverse directions with the
PCR primers described above. Sequencing was performed with an ABI PRISM 310 DNA Sequencer (Applied Biosystems, Foster City, CA). 


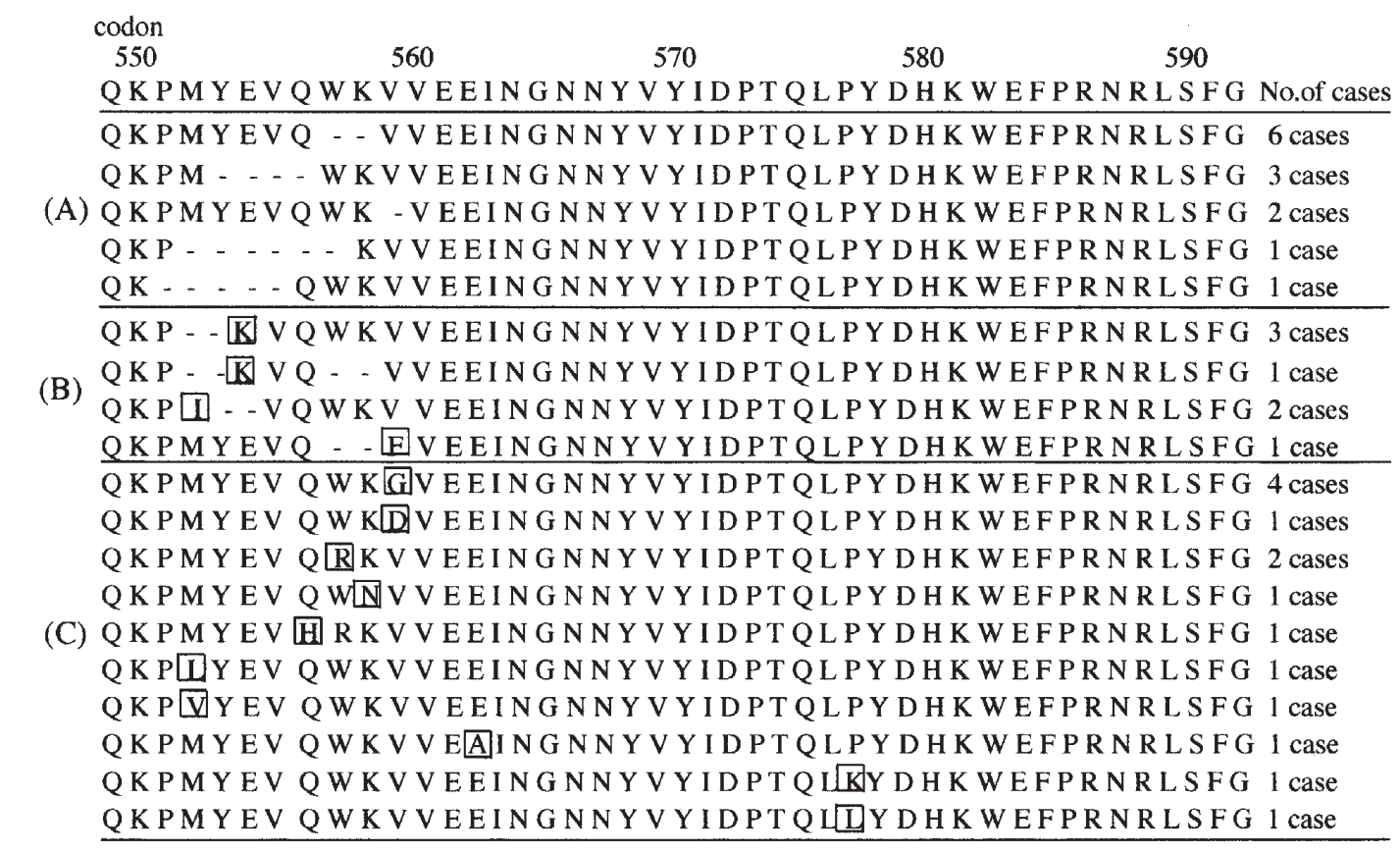

Figure 3. Predicted amino acid sequences of KIT with exon 11 mutations. The sequence starts at codon 549 and ends at 592 . The wild-type sequence is shown above. Blanks (---) correspond to deletions, and amino acid substitutions are shown in boxes ( $\square$ ). (A) Simple deletions detected in KIT exon 11; (B) Deletions and substitutions detected together in KIT exon 11; and (C) substitutions alone found in KIT exon 11.

Statistical methods. To evaluate the relationships between mutations and clinicopathological factors, data were analyzed by $\chi^{2}$ or Fisher's exact test in cross tables and by Student's t-test. Survival curves were drawn according to the KaplanMeier method and were compared by Fisher's exact test. The significance level was set at $5 \%$ for each analysis.

\section{Results}

Clinicopathological features. Of 56 selected GISTs, 39 (70\%) expressed both KIT and CD34, 12 (21\%) expressed only KIT, and 5 (9\%) expressed only CD34 (Fig. 1A and B). The patients from whom these GISTs were obtained comprised 35 men and 21 women, and the mean age of the patients at the time of surgical resection was 61 years (range 27-86 years). The mean tumor size was $4.4 \mathrm{~cm}$ (range 1-20 cm). During the follow-up period, liver metastases occurred in six patients, all of whom died of the disease within 4 years of surgery.

Evaluation of mutations. KIT mutations were detected in $36(64 \%)$ of 56 gastric GISTs. Thirty-four $(61 \%)$ of these mutations were in exon 11 , and $2(4 \%)$ were in exon 13 . We did not detect any mutations in exon 9 or exon 17 of KIT.

Examples of mutations detected in exon 11 of KIT are shown in Fig. 2A. Thirteen cases $(23 \%)$ showed simple deletions, 14 cases $(25 \%)$ showed substitutions, and 7 cases (13\%) showed substitutions and deletions, not always in the same order (Fig. 3). Deletions were comprised of 3-18 bp, and none disrupted the downstream open reading frame of the gene. In other words, all of the deletions in exon 11 observed in this study were in-frame deletions. The most common mutations in KIT exon 11 were a WK (Trp-Lys) deletion at codon 557 in 8 cases and a V559G substitution in 4 cases. In all cases, the deletions contained sequences between codons 550 and 560. Most of the substitutions also occurred in the same region of exon 11; however, a few substitutions were detected in other codons. Thus, most of the mutations in exon 11 of KIT were clustered at the $5^{\prime}$ end, which is a previously described 'hot spot' (2). Internal tandem duplications (ITDs) at the 3 ' end of exon 11 , which has also been described as a 'hot spot' (18), were not found in the study. In 29 (85\%) of the 34 GISTs with exon 11 mutations, we detected KIT wild-type, indicating that only 1 of the 2 KIT alleles was mutated in these GIST samples. Homozygous mutations were present in $5(15 \%)$ cases.

Two mutations were detected in exon 13 of KIT. Both were substitutions; one was Val643Ala and the other was Leu647Pro. The wild-type allele was not detected in either case, and therefore, both were considered to be homozygous (Fig. 2B).

Of 20 GISTs lacking KIT mutations, 7 cases $(35 \%)$ had PDGFRA mutations. Six cases were the same substitutions $\mathrm{D} 842 \mathrm{~V}$ in exon 18 which is the most common PDGFRA mutation in GISTs (Fig. 2C) (19), and the other one case was the E563K substitution in exon 12.

Correlation between clinicopathological and genetic features. The relationships between clinicopathological and genetic features of KIT are shown in Table I. KIT was expressed in $90 \%$ of KIT mutation-negative GISTs and in $92 \%$ of KIT mutation-positive GISTs. CD34 was expressed in 75 and $81 \%$, respectively. Liver metastases occurred in $6(17 \%)$ patients with KIT mutation-positive GIST after surgical resection, whereas liver metastasis occurred in none of the patients with KIT mutation-negative GIST; however, the difference was not statistically significant. KIT mutation-positive GISTs tended to be larger than KIT mutation-negative GISTs, and KIT mutation-positive GISTs tended to have a large Ki-67 LI (Fig. 1C). MVD assessed by CD31 and apoptotic index 
Table I. Correlation between clinicopathological and genetic features of KIT

\begin{tabular}{lccc}
\hline KIT mutation & Negative (\%) & Positive (\%) & p-value \\
\hline Number of cases & $20(36)$ & $36(64)$ & \\
KIT expression & $18(90)$ & $33(92)$ & 0.834 \\
CD34 expression & $15(75)$ & $29(81)$ & 0.627 \\
Sex (male/female) & $14 / 6$ & $21 / 15$ & 0.388 \\
Age, years (range) & $57(27-75)$ & $62(35-86)$ & 0.102 \\
Tumor size (cm) & 3.7 & 4.9 & 0.205 \\
Liver metastasis & $0 / 20(0)$ & $6 / 36(17)$ & 0.053 \\
Ki-67 LI (\%) & 1.6 & 2.5 & 0.155 \\
MVD & 14.3 & 15.2 & 0.717 \\
Apoptotic index (\%) & 0.58 & 0.61 & 0.810 \\
\hline
\end{tabular}

Ki-67 LI, Ki-67 labelling index; MVD, microvessel density.

assessed by TUNEL in tumors (Fig. 1D and E) showed no relation to KIT mutations.

We next classified the KIT mutations into 2 subtypes, deletions in the KIT gene and substitutions in the KIT gene, and we compared these groups and a KIT mutation-negative group with tumor size, frequency of liver metastases, Ki-67 LI, MVD and apoptotic index (Table II). A significant difference was detected in the frequency of liver metastases between the deletion mutations and KIT mutation-negative groups. A higher frequency of liver metastasis was observed in patients with deletion mutations than in KIT mutation-negative patients. With respect to tumor size and $\mathrm{Ki}-67 \mathrm{LI}$, these values tended to be largest in the deletion-mutant group, with lower values in the substitution group and the lowest values in the no KIT mutation group. However, the differences between these 3 groups were not statistically significant. We did not detect an association between MVD or apoptotic index and any of the groups.

Liver metastasis and death due to the original disease were not observed in the patients with KIT exon 13 mutations
Table II. Correlation between clinicopathological features and genetic subtypes of KIT.

Deletions Substitutions No KIT

mutation

\begin{tabular}{lccc}
\hline Number of cases & 20 & 16 & 20 \\
Tumor size $(\mathrm{cm})$ & 5.4 & 4.2 & 3.7 \\
Liver metastasis $(\%)$ & $5 / 20(25)^{\mathrm{a}}$ & $1 / 16(6)$ & $0 / 20(0)^{\mathrm{a}}$ \\
Ki-67 LI (\%) & 2.8 & 2.2 & 1.6 \\
MVD & 17.6 & 12.4 & 14.3 \\
Apoptotic index (\%) & 0.67 & 0.47 & 0.61 \\
\hline
\end{tabular}

Ki-67 LI, Ki-67 labelling index; MVD, microvessel density. ${ }^{\mathrm{a}} \mathrm{p}<0.05$.

during the follow-up period. In addition, there were no differences between heterozygous tumors and homozygous tumors with respect to liver metastasis or death due to the original disease (data not shown).

The clinicopathological features of GISTs with PDGFRA mutations are shown in Table III. Six patients were male and one was female. Age, tumor size, Ki-67 LI, MVD and apoptotic index were not statistically different compared to those of other groups.

Clinical follow-up. Clinical follow-up was available for all 56 cases, and the median follow-up period for survivors was 56.3 months (range 1-224 months). All 6 patients who experienced liver metastasis during the follow-up period died within 4 years after their original surgery. Kaplan-Meier analysis revealed a strong trend that the survival rate for patients whose tumors expressed a mutant KIT isoform was poorer than that for patients with KIT mutation-negative tumors (Fig. 4A), although there was no statistically significant association of KIT mutations with the rate of death from the original disease $(\mathrm{p}=0.053)$.

Overall survival was also analyzed and compared between the 3 groups; KIT deletions, KIT substitutions and no KIT

Table III. Clinicopathological data of GISTs with PDGFRA mutations.

\begin{tabular}{lccccccc}
\hline No. & Mutation & Sex & Age (years) & Size (cm) & Ki-67 LI (\%) & MVD & Apoptotic index (\%) \\
\hline 1 & D842V & M & 60 & 2.5 & 1.2 & 16 & 0.3 \\
2 & D842V & M & 59 & 4.5 & 1.4 & 20 & 0.2 \\
3 & D842V & M & 65 & 2.0 & 1.6 & 14 & 2.4 \\
4 & D842V & M & 63 & 1.6 & 0.4 & 10 & 0.4 \\
5 & D842V & M & 62 & 2.3 & 4.4 & 14 & 1.4 \\
6 & D842V & M & 57 & 6.0 & 1.3 & 17 & 0.2 \\
7 & E563K & F & 66 & 3.0 & 0.8 & 4 & 0.2 \\
\hline
\end{tabular}

PDGFRA, platelet-derived growth factor receptor $\alpha$; Ki-67 LI, Ki-67 labelling index; MVD, microvessel density. 

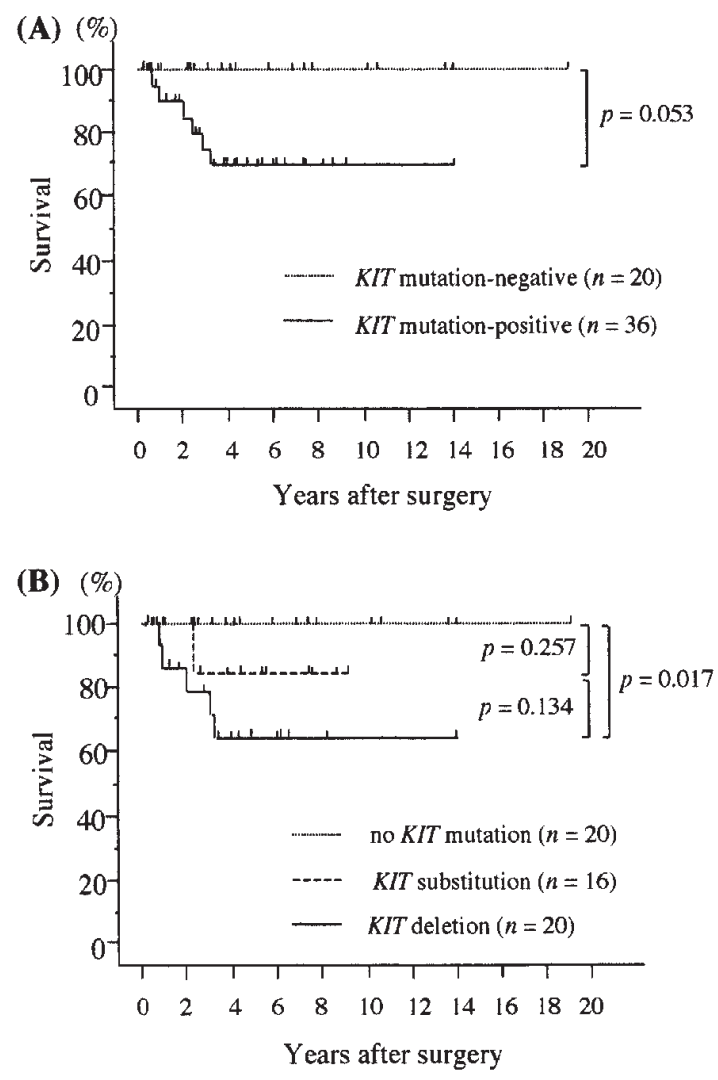

Figure 4. Relation between KIT mutations and survival rate. Postoperative survival curves were drawn according to the Kaplan-Meier method. The pvalues were calculated with Fisher's exact test. (A) The probability of overall survival for patients with KIT mutation-positive and KIT mutationnegative tumors. (B) The probability of overall survival for patients with deletion, substitution, and no mutation in the KIT gene.

mutations (Fig. 4B). Patients with tumors that harbored KIT deletions had a significantly poorer survival rate than those with no KIT mutations ( $\mathrm{p}=0.017$ ). There were no statistically significant differences in survival rate between KIT deletions and KIT substitutions or between KIT substitutions and no KIT mutations.

\section{Discussion}

The KIT gene encodes the receptor tyrosine kinase KIT, which is composed of an intracellular tyrosine kinase, a juxtamembrane (JM) region and an extracellular domain with a ligand-binding site. The KIT ligand, stem cell factor (SCF), binds to the KIT receptor, leading to receptor dimerization, activation of kinase activity and autophosphorylation (20). KIT mutations that undergo this autophosphorylation without the KIT ligand can contribute to the growth of GISTs (5).

The percentage of GISTs with KIT mutations has varied widely in previous studies. The mutations vary from single bp substitutions to complex deletions/insertions but are invariably in-frame mutations. Most have been found in the JM domain, especially between codons 550-560, which is the 'hot-spot' region of exon 11 (2). Taniguchi et al (14) reported that exon 11 mutations were present in $71(57 \%)$ of 124 cases, and Lasota et al (21) reported exon 11 mutations in $103(52 \%)$ of 200 cases. Other investigators have found exon 11 mutations at much lower rates $(<40 \%)(22,23)$. In the present study, we detected KIT exon 11 mutations in 61\% (34/56) of gastric GISTs. There are some possible reasons for the variations in KIT mutation rate. One possibility is that the diagnostic criteria for GIST were not consistent across the studies. It is also possible that the locations of GISTs, such as stomach, small intestine, or other sites, were not considered separately. Lastly, the method of DNA extraction from paraffin-embedded tissue or frozen tissue could affect detection of KIT mutations.

The frequency of KIT exon 9 mutations appeared to be much lower than that of exon 11 mutations. As previously reported, exon 9 mutations consist of identical tandem repeats of sequences encoding Ala-Tyr, which correspond to codons 502 and $503(21,24)$. Most tumors with exon 9 mutations were located in the small intestine. Kim et al (15) reported eight cases of GIST with mutations in exon 9, and only two of these cases were localized in the stomach. Hirota et al (25) reported seven cases of GIST with mutations in exon 9, and only one case was localized in the stomach. In the present study, we only examined GISTs localized in the stomach, and none of our samples carried a mutation in exon 9 of KIT.

In the present study, a mutation in exon 13 of KIT was found in 2 cases. Mutations in exon 13 of the KIT gene in GISTs are thought to be rare. To our knowledge, there are fewer than 10 reported sporadic GIST cases with mutations in exon 13, and all exhibit the same Lys642Glu substitution $(21,24,26)$. Chen et al $(27)$ reported a new coincident mutation in exon 13, Val654Ala, in STI571-resistant GISTs after treatment with STI571. In the present study, we detected two substitutions in exon 13, Val643Ala and Leu647Pro, in GISTs that were not treated with STI571. These substitutions were near the previously reported Lys642Glu site.

It has also been reported that mutations in exon 17 of KIT are much more rare in GISTs $(14,26)$. In the present study, no patient carried a mutation in exon 17 . The present data suggest that, in gastric GISTs, the KIT mutation rates in exons 9,13 and 17 are very low in comparison to that of exon 11. Therefore, when we evaluate the associations of genetic features of GISTs with clinicopathological features, it is essential to screen for mutations in exon 11 of KIT.

We compared clinicopathological features of KIT mutationpositive and KIT mutation-negative GIST and those of mutation subtypes. Previous studies suggested that KIT mutations are more common in malignant than in benign GISTs $(2,6,14-16)$, and our present results support these findings. We compared clinical outcome between KIT mutation-positive and KIT mutation-negative GISTs and found more frequent relapses of liver metastases and subsequent death due to the disease in patients with KIT mutation-positive GISTs. To be exact, 6 of 36 patients with KIT mutation-positive gastric GISTs died from the original disease during the follow-up period.

Furthermore, differences between mutation subtypes were detected. Singer et al (16) reported that patients with tumors with substitutions in exon 11 had a more favorable relapse-free survival rate than those with other mutations. However, another group found no relation between relapse-free survival rate and KIT subtypes (15). In the present study, deletions in exon 11 of KIT were associated with more malignant features than substitutions, and patients with such mutations were more likely to experience liver metastasis and die. Therefore, 
mutations in the KIT gene, especially deletions, may serve as a genotypic marker that correlates with malignancy.

$\mathrm{Ki}-67$ is one of the most reliable prognostic factors for gastric GISTs (28). A high Ki-67 LI is considered an independent predictor of poor prognosis. In the present study, Ki-67 LI tended to be higher in GISTs with KIT mutations than in those without KIT mutations. Furthermore, when KIT mutations were classified into 2 subtypes, deletions and substitutions, the Ki-67 LI was highest in GISTs with deletions, lower in GISTs with substitutions, and lowest in GISTs without any KIT mutations. However, these differences were not statistically significant. In contrast, MVD, which is a measure of angiogenesis and is considered a strong indicator of prognosis in patients with a variety of cancers, did not show any relationship with KIT mutation-positive or KIT mutation-negative GIST or presence or absence of deletions. The growth pathway of GISTs localized in the stomach may differ from that of other gastric cancers, and the mechanism remains to be clarified. We also examined cell apoptosis by TUNEL; we did not observe any differences between KIT mutation-positive and KIT mutation-negative GISTs.

Recently, it has been reported that a subset of GISTs had mutation in the other site. Heinrich et al (7) reported that $35 \%$ of GISTs lacking KIT mutations had activating mutations in the PDGFRA and that mutations in PDGFRA and KIT were mutually exclusive. In the present study, 7 out of 20 cases of KIT wild-type GISTs had mutations in the PDGFRA gene.

In conclusion, we have shown that KIT mutations, especially deletions which we detected only in exon 11, are associated with occurrence of liver metastasis after complete tumor resection and subsequent death due to the original disease. Post-surgical treatment with STI571 and precise follow-up after surgery may improve the prognosis of patients with a localized gastric GIST with a KIT mutation, especially deletion in exon 11.

\section{References}

1. Miettinen M, Sarlomo-Rikala M and Lasota J: Gastrointestinal stromal tumors: recent advances in understanding of their biology. Hum Pathol 30: 1213-1220, 1999.

2. Lasota J, Jasinski M, Sarlomo-Rikala M and Miettinen M: Mutations in exon 11 of $c$-Kit occur preferentially in malignant versus benign gastrointestinal stromal tumors and do not occur in leiomyomas or leiomyosarcomas. Am J Pathol 154: 53-60, 1999.

3. Miettinen M and Lasota J: Gastrointestinal stromal tumors definition, clinical, histological, immunohistochemical, and molecular genetic features and differential diagnosis. Virchows Arch 438: 1-12, 2001.

4. Dematteo RP, Heinrich MC, El-Rifai WM and Demetri G: Clinical management of gastrointestinal stromal tumors: before and after STI-571. Hum Pathol 33: 466-477, 2002.

5. Hirota S, Isozaki K, Moriyama Y, et al: Gain-of-function mutations of $c$-kit in human gastrointestinal stromal tumors. Science 279: 577-580, 1998.

6. Hou YY, Tan YS, Sun MH, et al: C-kit gene mutation in human gastrointestinal stromal tumors. World J Gastroenterol 10 : 1310-1314, 2004.

7. Heinrich MC, Corless CL, Duensing A, et al: PDGFRA activating mutations in gastrointestinal stromal tumors. Science 299: 708-710, 2003.
8. Druker BJ, Talpaz M, Resta DJ, et al: Efficacy and safety of a specific inhibitor of the BCR-ABL tyrosine kinase in chronic myeloid leukemia. N Engl J Med 344: 1031-1037, 2001.

9. Druker BJ, Sawyers CL, Kantarjian H, et al: Activity of a specific inhibitor of the BCR-ABL tyrosine kinase in the blast crisis of chronic myeloid leukemia and acute lymphoblastic leukemia with the Philadelphia chromosome. N Engl J Med 344: 1038-1042, 2001 .

10. Joensuu H, Roberts PJ, Sarlomo-Rikala M, et al: Effect of the tyrosine kinase inhibitor STI571 in a patient with a metastatic gastrointestinal stromal tumor. N Engl J Med 344: 1052-1056, 2001 .

11. Van Oosterom A, Judson I, Verweij J, et al: Safety and efficacy of imatinib (STI571) in metastatic gastrointestinal stromal tumors: a phase I study. Lancet 358: 1421-1423, 2001.

12. Demetri GD, von Mehren M, Blanke CD, et al: Efficacy and safety of imatinib mesylate in advanced gastrointestinal stromal tumors. N Engl J Med 347: 472-480, 2002.

13. Heinrich MC, Corless CL, Demetri GD, et al: Kinase mutations and imatinib response in patients with metastatic gastrointestinal stromal tumor. J Clin Oncol 21: 4342-4349, 2003.

14. Taniguchi M, Nishida T, Hirota S, et al: Effect of $c$-kit mutation on prognosis of gastrointestinal stromal tumors. Cancer Res 59: 4297-4300, 1999.

15. Kim TW, Lee H, Kang YK, et al: Prognostic significance of c-kit mutation in localized gastrointestinal stromal tumors. Clin Cancer Res 10: 3076-3081, 2004.

16. Singer S, Rubin BP, Lux ML, et al: Prognostic value of KIT mutation type, mitotic activity, and histologic subtype in gastrointestinal stromal tumors. J Clin Oncol 20: 3898-3905, 2002.

17. Rubin BP, Singer S, Tsao C, et al: KIT activation is a ubiquitous feature of gastrointestinal stromal tumors. Cancer Res 61: 8118-8121, 2001.

18. Antonescu CR, Sommer G, Sarran L, et al: Association of KIT exon 9 mutations with nongastric primary site and aggressive behavior: KIT mutation analysis and clinical correlates of 120 gastrointestinal stromal tumors. Clin Cancer Res 9: 3329-3337, 2003.

19. Corless CL, Schroeder A, Griffith D, et al: PDGFRA mutations in gastrointestinal stromal tumors: frequency, spectrum and in vitro sensitivity to imatinib. J Clin Oncol 23: 1-8, 2005.

20. Vosseller K, Stella G, Yee NS and Besmer P: c-kit receptor signaling through its phosphatidylinositide-3'-kinase-binding site and protein kinase $\mathrm{C}$ : role in mast cell enhancement of degranulation, adhesion, and membrane ruffling. Mol Biol Cell 8: 909-922, 1997.

21. Lasota J, Wozniak A, Sarlomo-Rikala M, et al: Mutations in exon 9 and exon 13 of KIT gene are rare events in gastrointestinal stromal tumors. A study of 200 cases. Am J Pathol 157: 1091-1095, 2000.

22. Ernst SI, Hubbs AE, Przygodzki RM, Emory TS, Sobin LH and O'Leary TJ: KIT mutation portends poor prognosis in gastrointestinal stromal/smooth muscle tumors. Lab Invest 78 : 1633-1636, 1998 .

23. Moskaluk CA, Tian Q, Marshall CR, Rumpel CA, Franquemont DW and Frierson HF Jr: Mutations of $c$-kit JM domain are found in a minority of human gastrointestinal stromal tumors. Oncogene 18: 1897-1902, 1999.

24. Lux ML, Rubin BP, Biase TL, et al: KIT extracellular and kinase domain mutations in gastrointestinal stromal tumors. Am J Pathol 156: 791-795, 2000.

25. Hirota S, Nishida T, Isozaki K, et al: Gain-of-function mutation at the extracellular domain of KIT in gastrointestinal stromal tumours. J Pathol 193: 505-510, 2001

26. Kinoshita K, Isozaki K, Hirota S, et al: c-kit gene mutation at exon 17 or 13 is very rare in sporadic gastrointestinal stromal tumors. J Gastroenterol Hepatol 18: 147-151, 2003.

27. Chen LL, Trent JC, Wu EF, et al: A missense mutation in KIT kinase domain 1 correlates with imatinib resistance in gastrointestinal stromal tumors. Cancer Res 64: 5913-5919, 2004.

28. Wong NA, Young R, Malcomson RD, et al: Prognostic indicators for gastrointestinal stromal tumours: a clinicopathological and immunohistochemical study of 108 resected cases of the stomach. Histopathology 43: 118-126, 2003. 